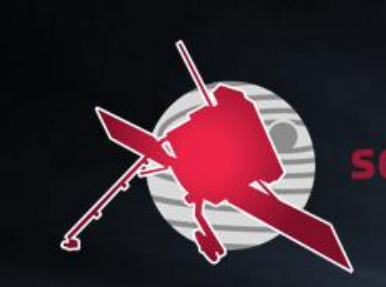

\title{
Stray light calibration for the Solar Orbiter/Metis solar coronagraph
}

- $\quad$ F. Landinia $^{a}$, M. Romoli ${ }^{b}$, S. Fineschic ${ }^{c}$ C. Casini ${ }^{b}$, C. Baccani ${ }^{a}$, E. Antonuccic, G. Nicolinic, G. Naletto ${ }^{\text {d,e }}$, P. Nicolosif ${ }^{f, e}$, D. Spadarog, V. Andretta ${ }^{\text {h }}$, M. Castronuovo', M. Castic, G.

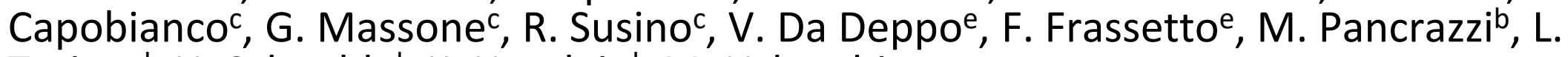
Teriaca', U. Schuehle', K. Heerlein', M. Uslenghim

- $\quad$ aINAF - Osservatorio Astrofisico di Arcetri, Italy;

- $\quad$ bipartimento di Fisica e Astronomia - Università degli Studi di Firenze, Italy;

- $\quad$ INAF - Osservatorio Astrofisico di Torino, Italy;

- $\quad$ UUniversity of Padova, Department of Physics and Astronomy, Italy;

- $\quad$ eCNR - IFN, Italy;

- fDipartimento di Ingegneria dell'Informazione, Università di Padova, Italy;

- $\quad$ gINAF - Osservatorio Astrofisico di Catania, Italy;

- $\quad$ hINAF - Osservatorio Astronomico di Capodimonte, Napoli, Italy;

- $\quad$ iASI, Roma, Italy;

- IMax Planck Institute for Solar System Research, Germany;

- mINAF - IASF, Milano, Italy 


\section{Outline}

- Solar Orbiter/Metis

- General introduction

- Instrument description

- Calibration facility and setup

- Stray light calibrations data analysis flow

- Stray light calibration results

- IO alignment

- Different heliocentric distances

- Off-pointing

- Conclusions 


\section{Metis}

-Polarized VL imaging @ 580 - 640 nm

- UV HI Ly $\alpha$ imaging @ $121.6 \pm 10$ nm

- $\operatorname{FoV}\left(1.5^{\circ} \cdot 2.9^{\circ}\right.$ annular, 1.6-3.0 $\left.\mathrm{R}_{\square} @ 0.28 \mathrm{AU}\right)$

- Spatial sampling element $\leq 4000$ km (20") @ $0.28 \mathrm{AU}$

- Time resolution $\geq 1 \mathrm{sec}$

- Simultaneous VL and UV imaging

Units:

MOU METIS Optical Unit (boom and telescope) MPPU METIS Power Processing Unit HVU High Voltage Unit CPC Camera Power Converter

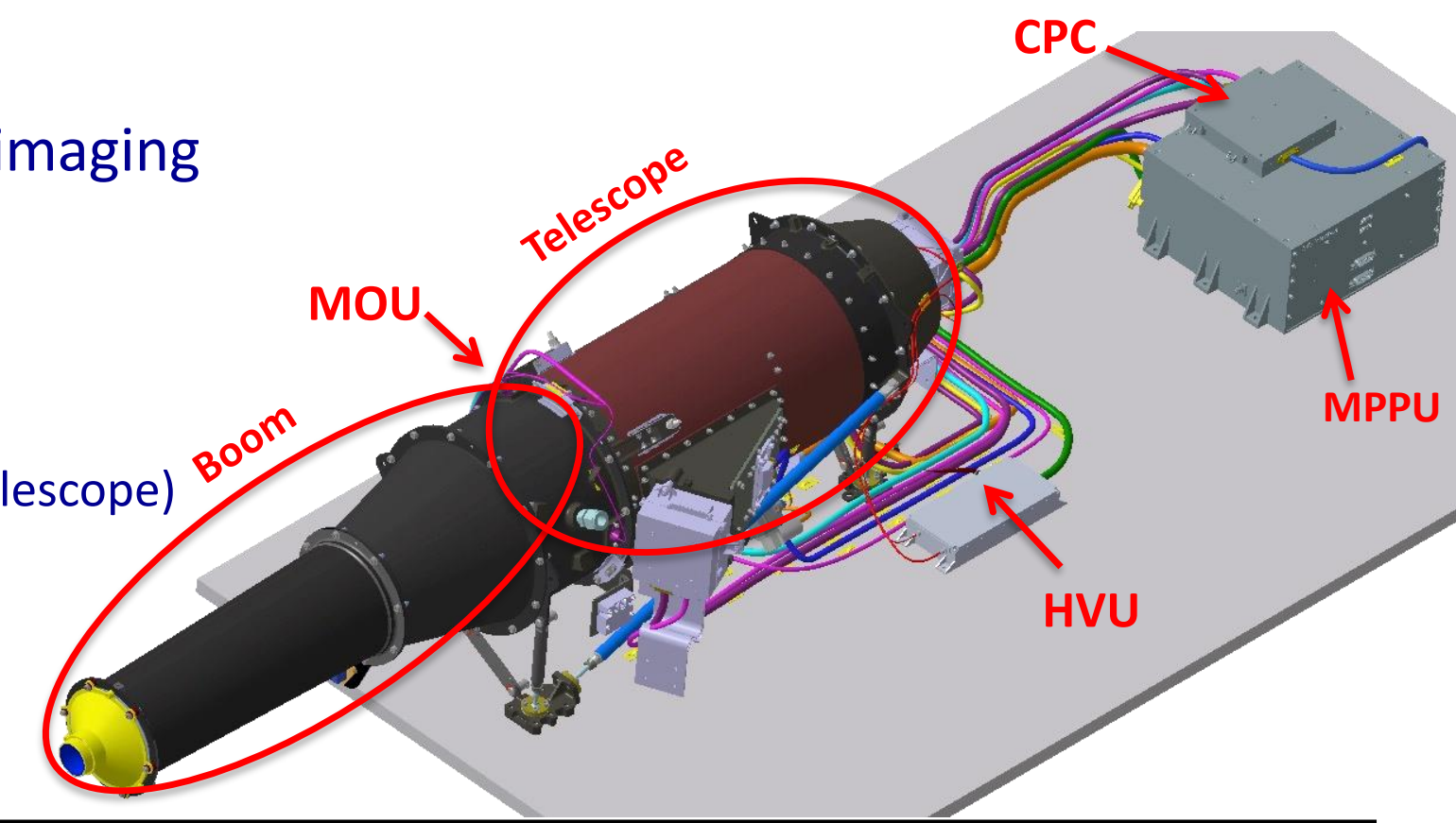




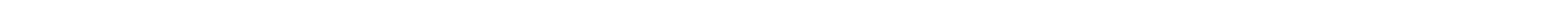




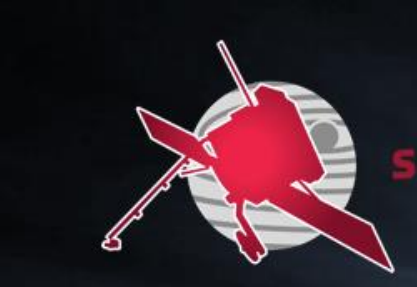

Inverted

external occulter

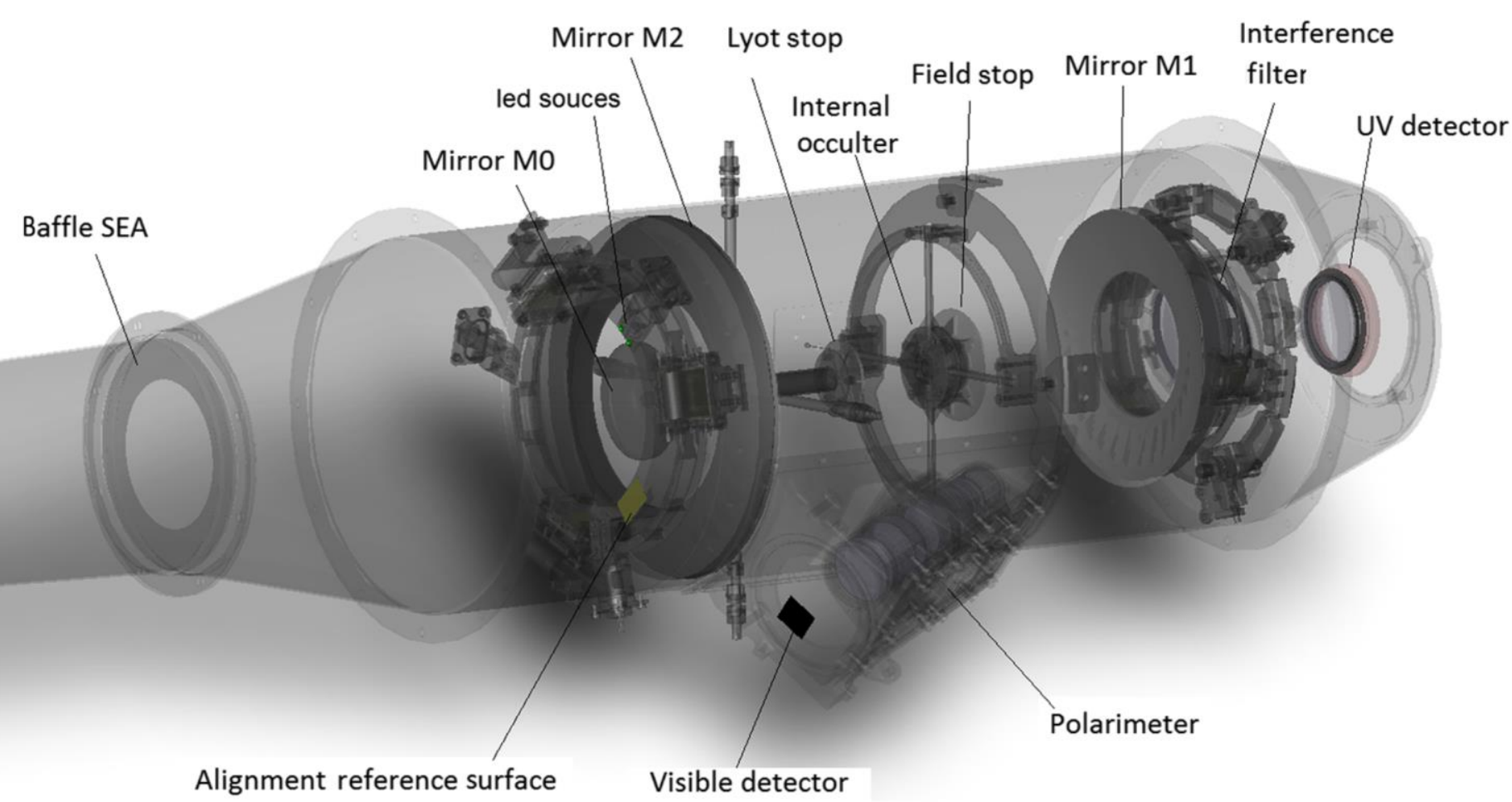




\section{On ground calibrations}

- AIT/AIV and calibrations were performed at the OPSys (Optical Payload System) facility, an INAF laboratory hosted by ALTEC S.p.A. in Torino.

- OPSys: three communicating clean rooms which host the SPOCC (Space Optics Calibration Chamber)

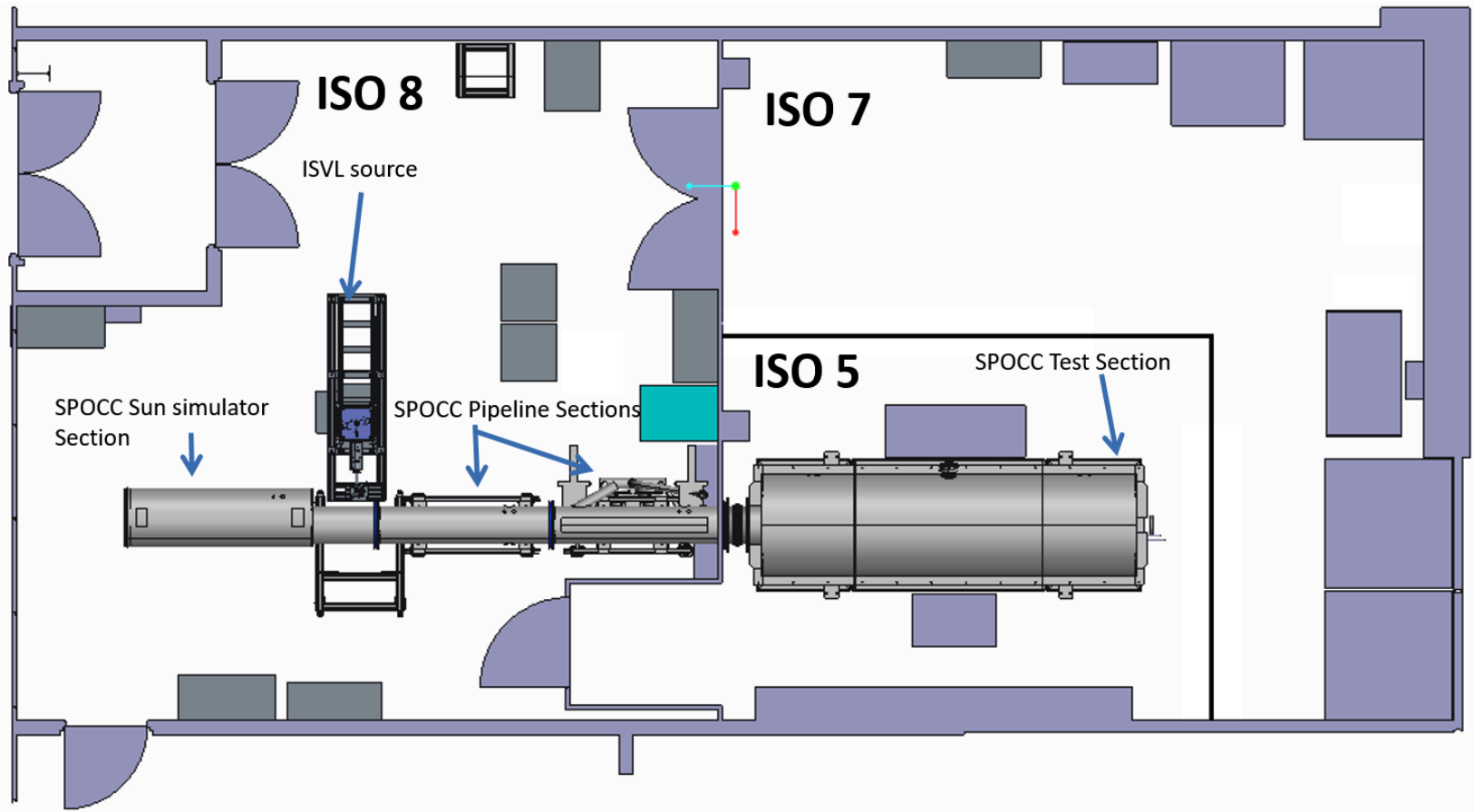

- $\quad$ SPOCC is a solar divergence simulator that can operate in air and in vacuum. 


\section{Stray light calibration}

\section{IIn}

- The most stringent requirement to be verified, as far as stray light is concerned, is in the visible light range:

- \# METIS-IRD-REQ-122: $B_{\text {stray }}<10^{-9} B_{\text {sun }}$

Where $B_{\text {stray }}$ is the stray light irradiance on the focal plane and $B_{\text {sun }}$ is the mean solar disk irradiance as imaged on the Metis focal plane in absence of occulting system.

- SPOCC (Space Optics Calibration Chamber) with ISVL (Illumination System for Visible-light) source: a stabilized arc lamp designed to simulate an irradiance profile similar to the photosphere (limb darkening included).

- On ground calibrations offer a unique chance for obtaining a stray light image isolated with respect to the coronal signal. 


\section{Data analysis flow}

Needed images \& parameters

- Ingredients

SL raw image

Exp DT1 sec

Background

Exp DT1 sec

Stray light image and relative dark
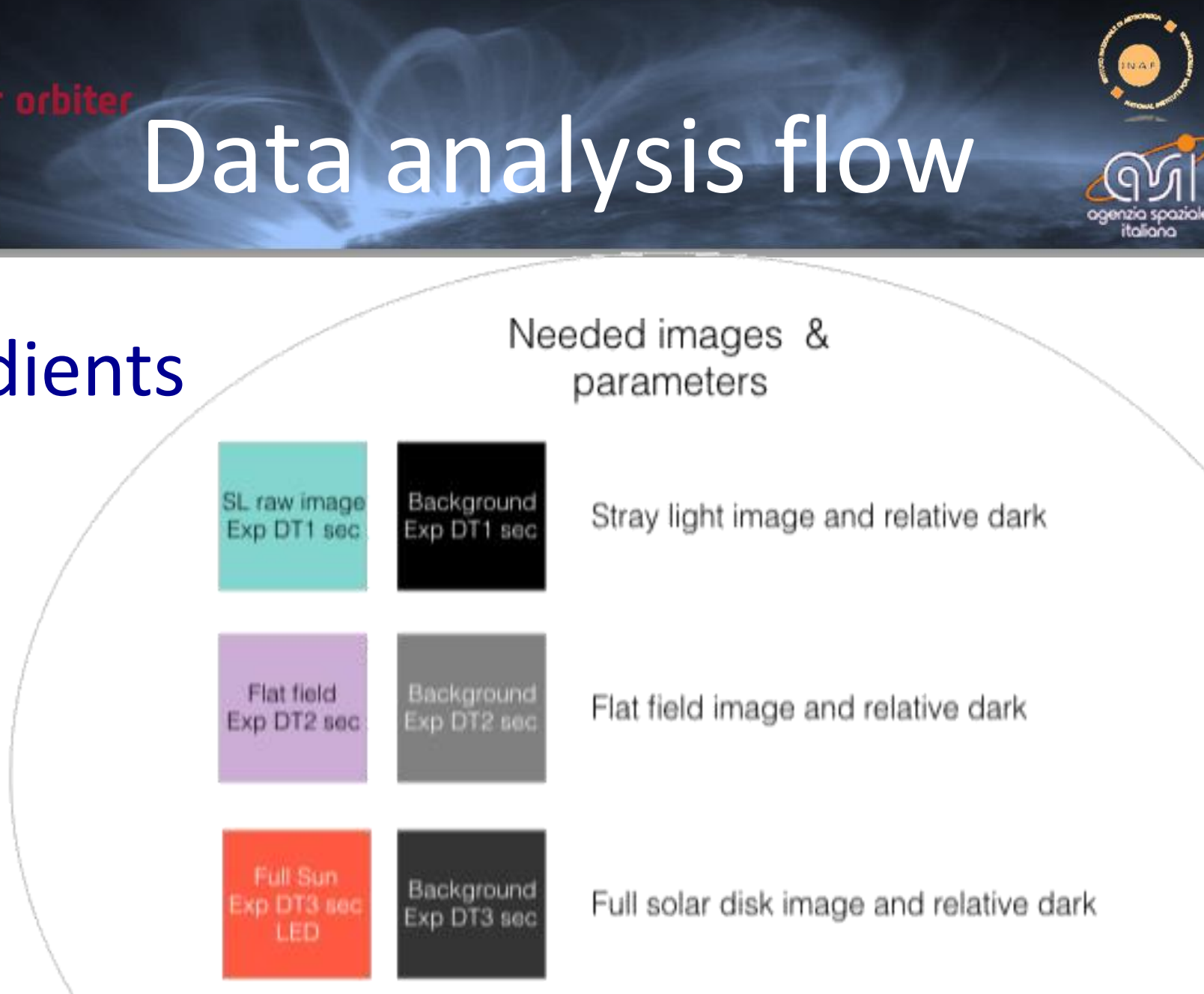

Flat field image and relative dark

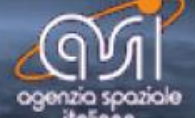

PD ISVL Photodiode measurement of LED solar disk

Full solar disk image and relative dark 

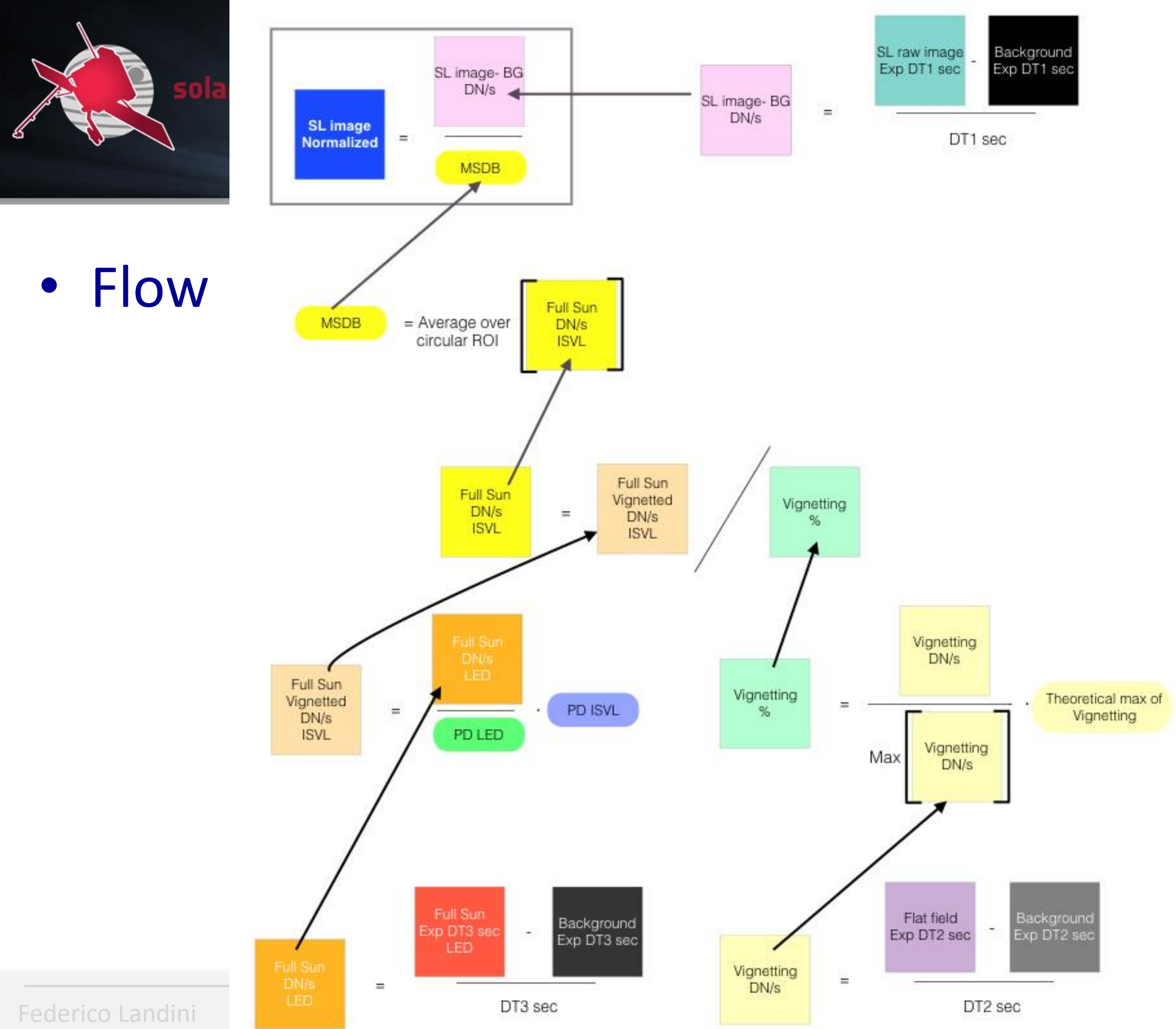


\section{Solar disk image}

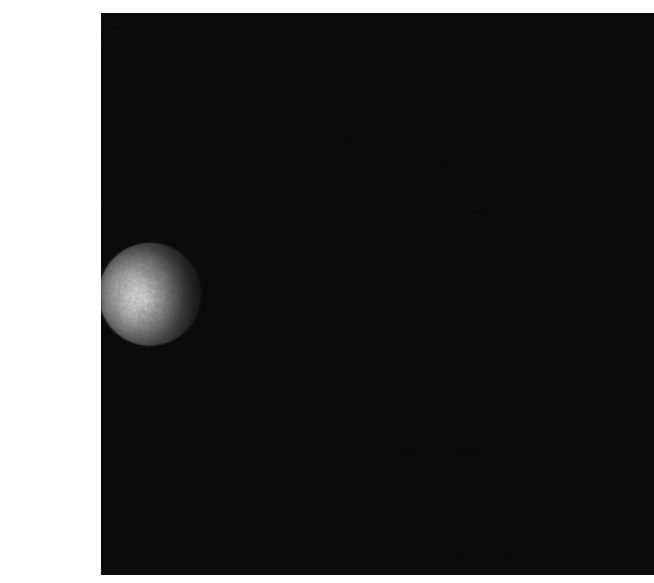

MSDB (DN/s) = Average of the signal in the red ROI 
Mvean solar disk brightness

\section{(0) \\ (MEDB)}

므믄

- The ISVL solar disk saturates the VL detector even at the minimum exposure time.

- An alternative decentered solar disk image is taken with a much dimmer LED source.

- Measurement of the solar disk irradiance in both cases are taken with a calibrated photodiode.

- Finally, a proportion is computed to retrieve the MSDB as it should be with the ISVL source. 
Stray light at $0.5 \mathrm{AU}$ before 10 position

\section{finetuning}

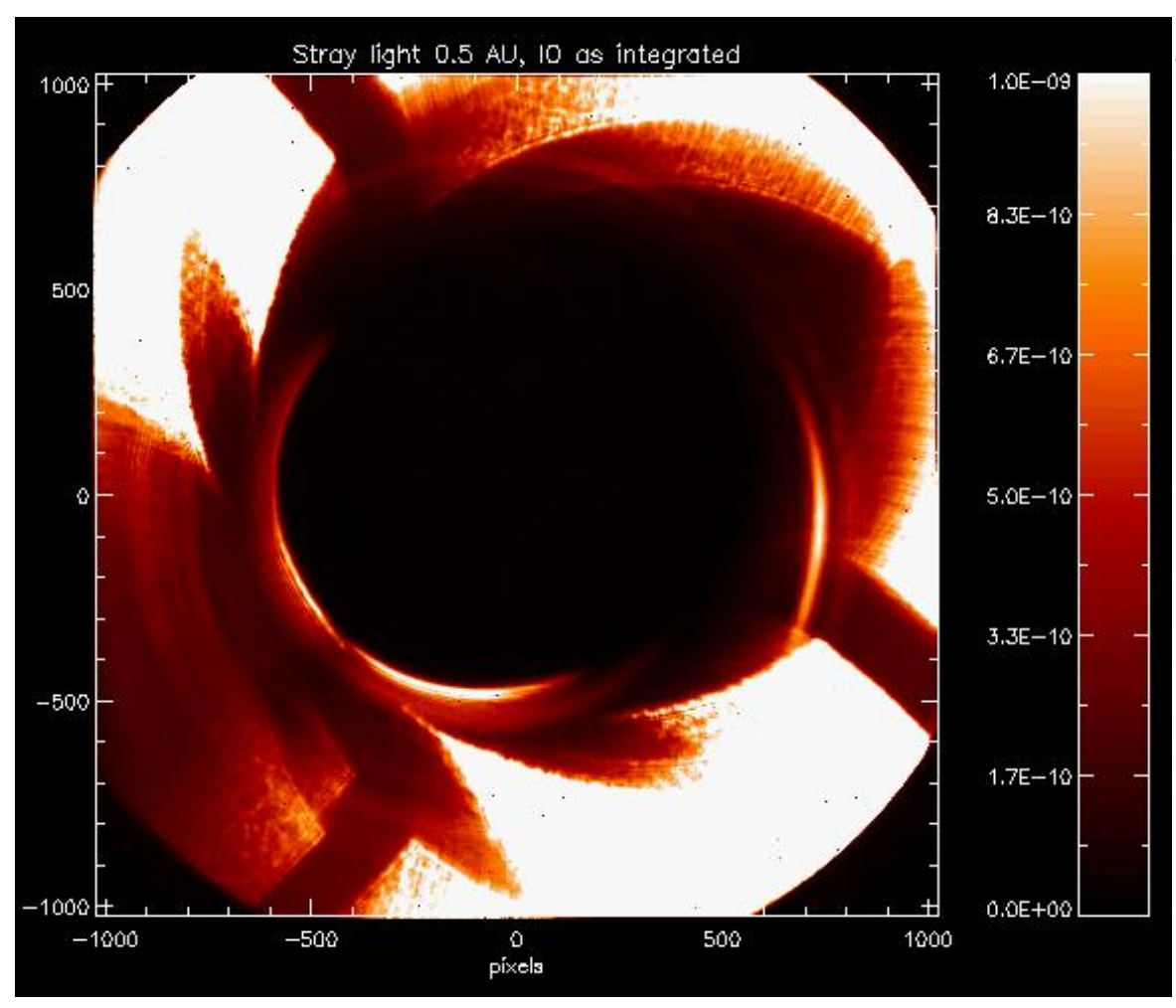

Acquired image

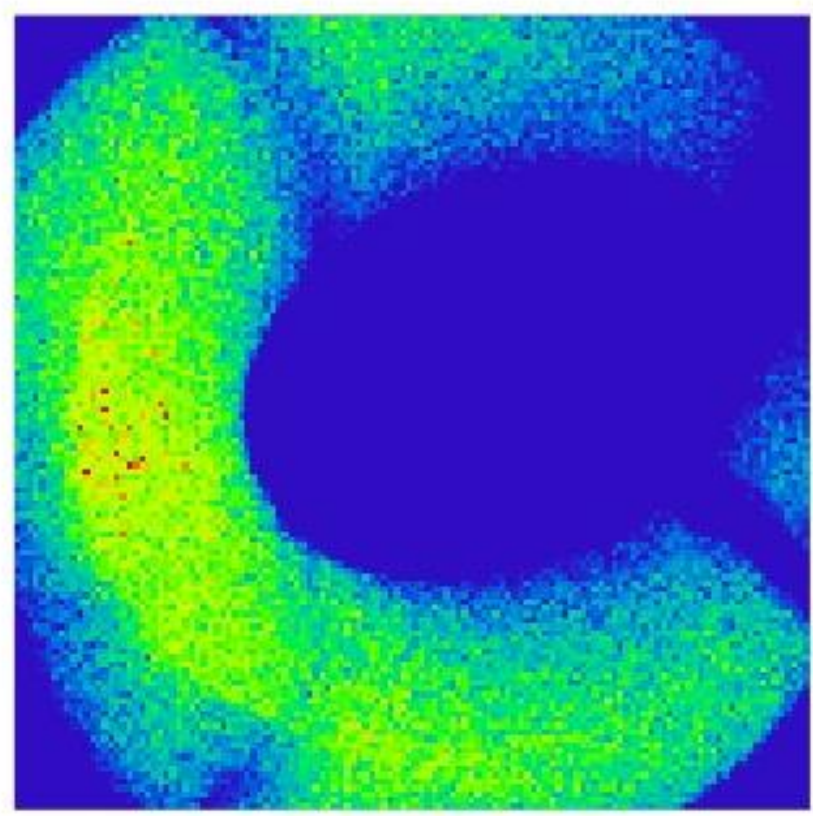

बजा

metis

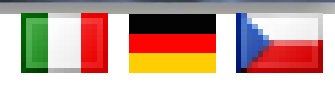

Zemax simulation with offcentered IO 


\section{Quantitative 10 adjusting}

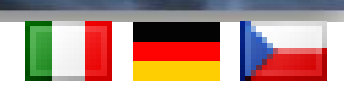

- ROls have been selected in order to encompass the image of the IO edge and are relative to the 4 sectors defined by the two motors orientation and direction.

- The ROI signal average is evaluated for each IO position.
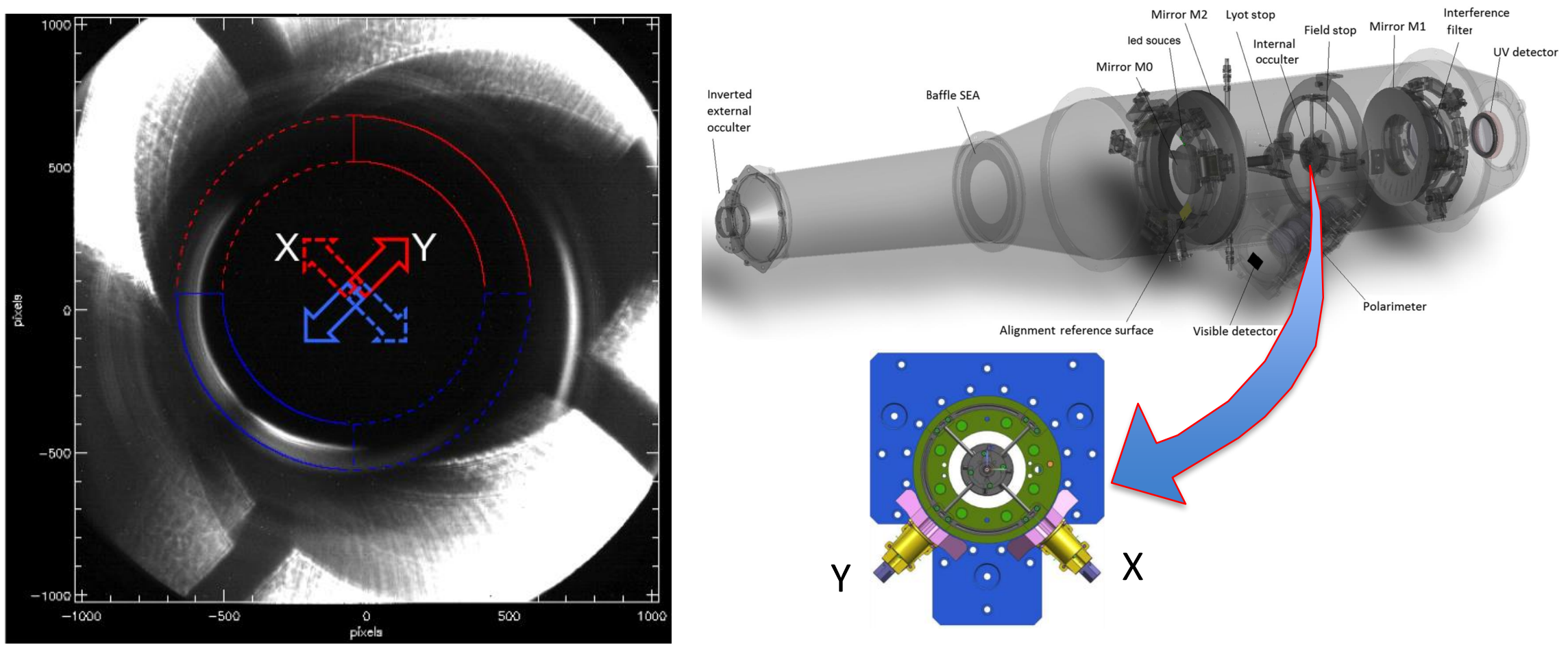


\section{Quantitative 10 adjusting}
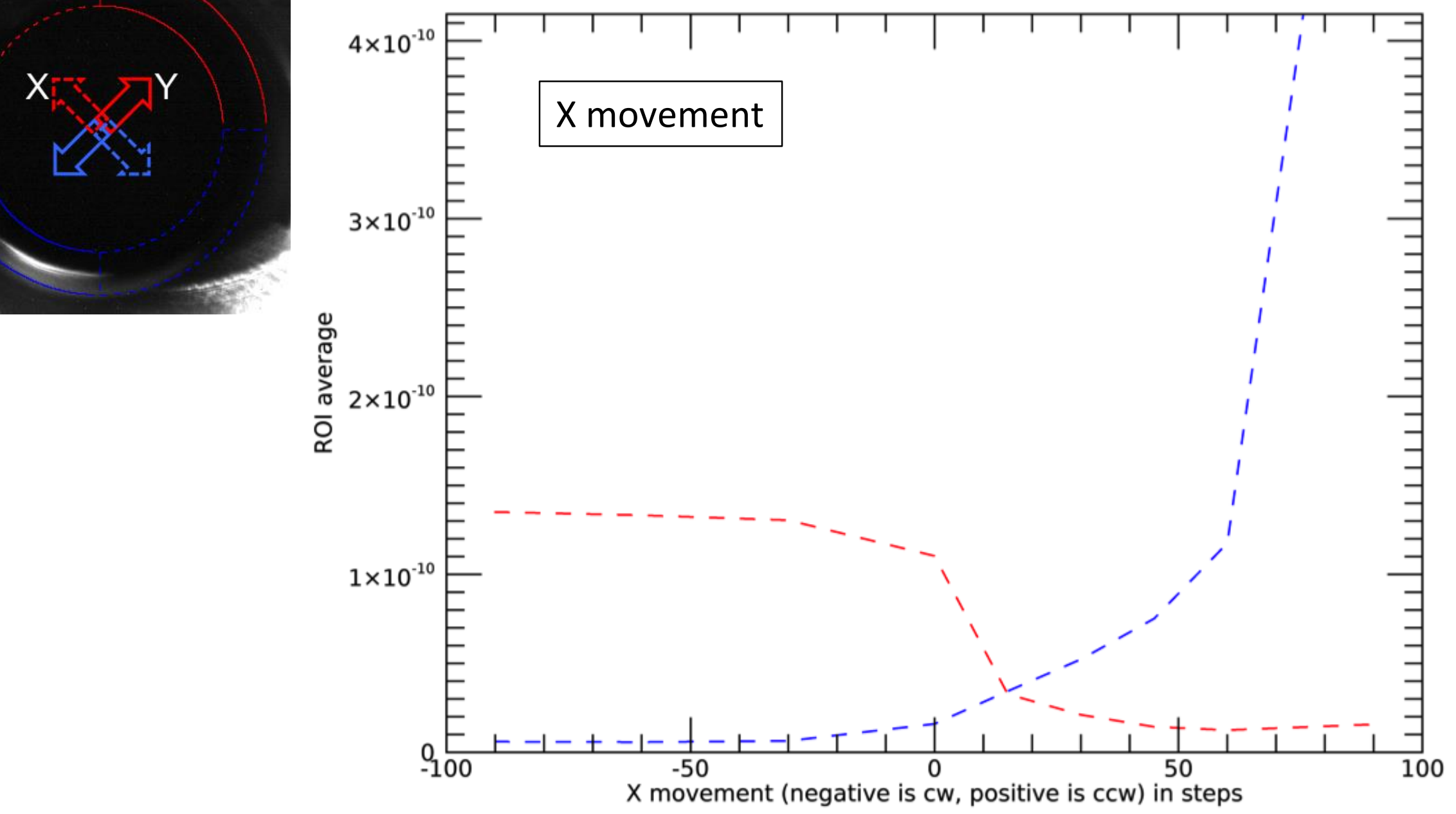


\section{Quantitative 10 adjusting}
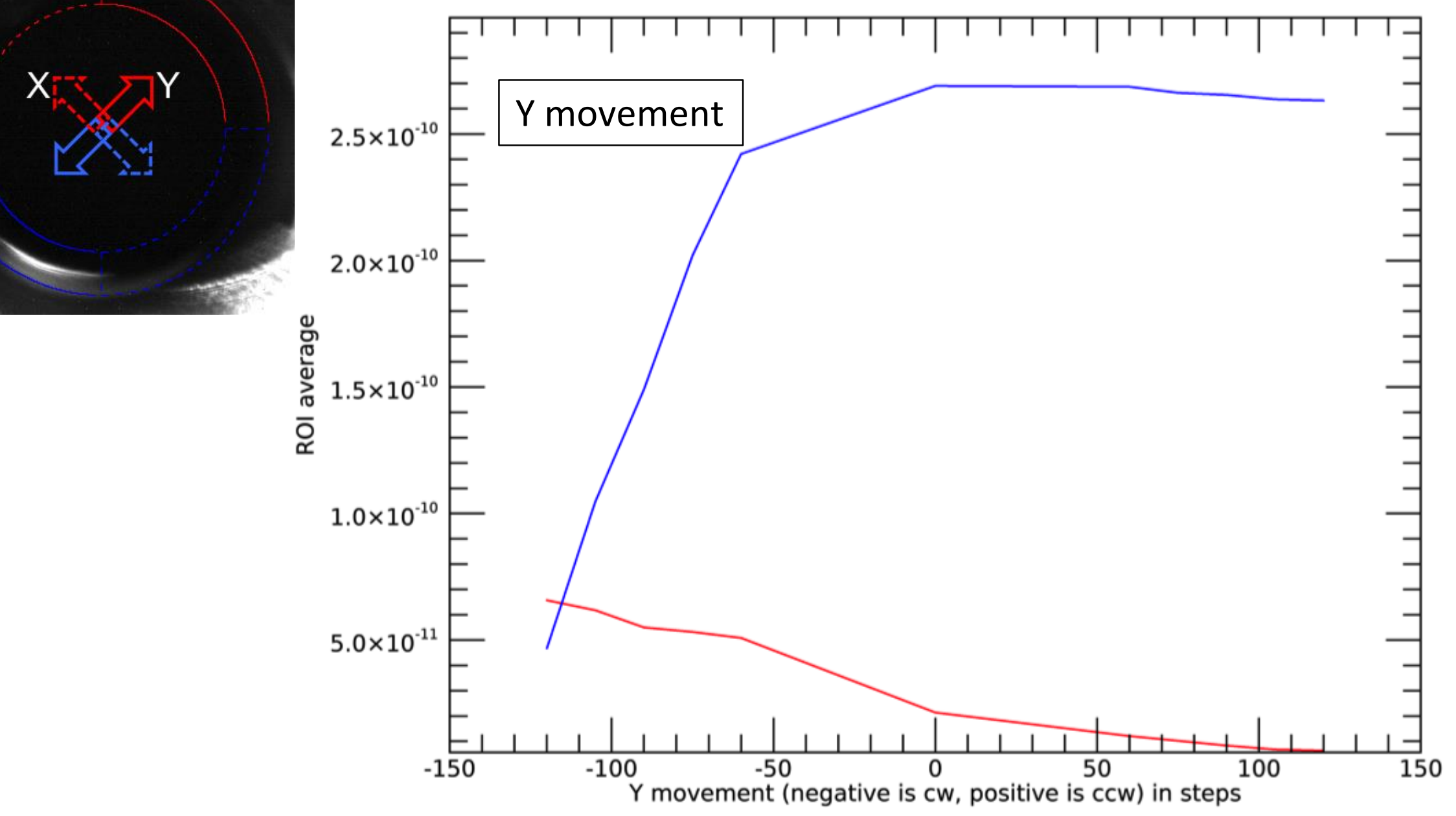

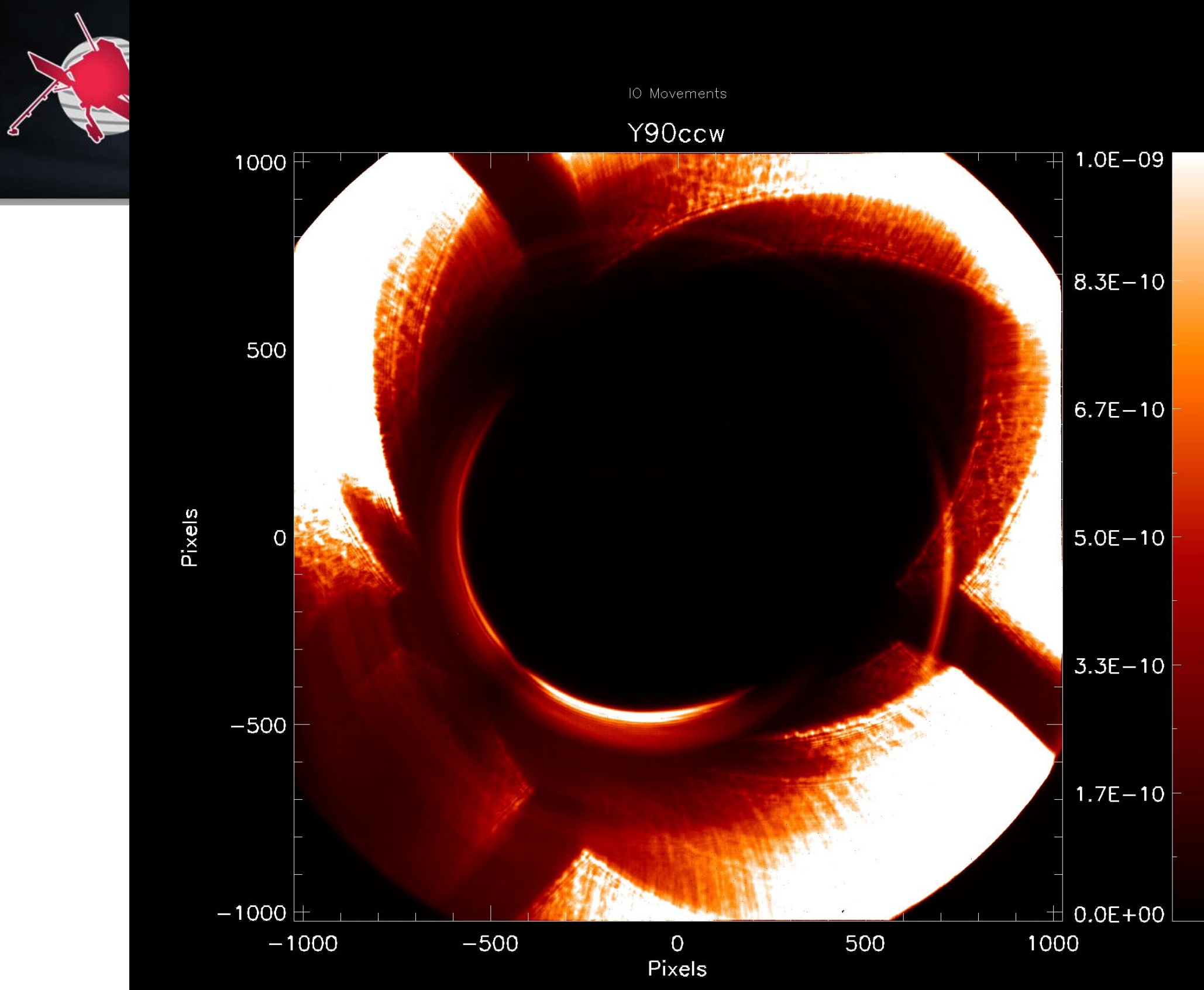


\section{Stray light 0.5 AU}

0

Stray light $0.5 \mathrm{AU}, 10$ position fine tuned

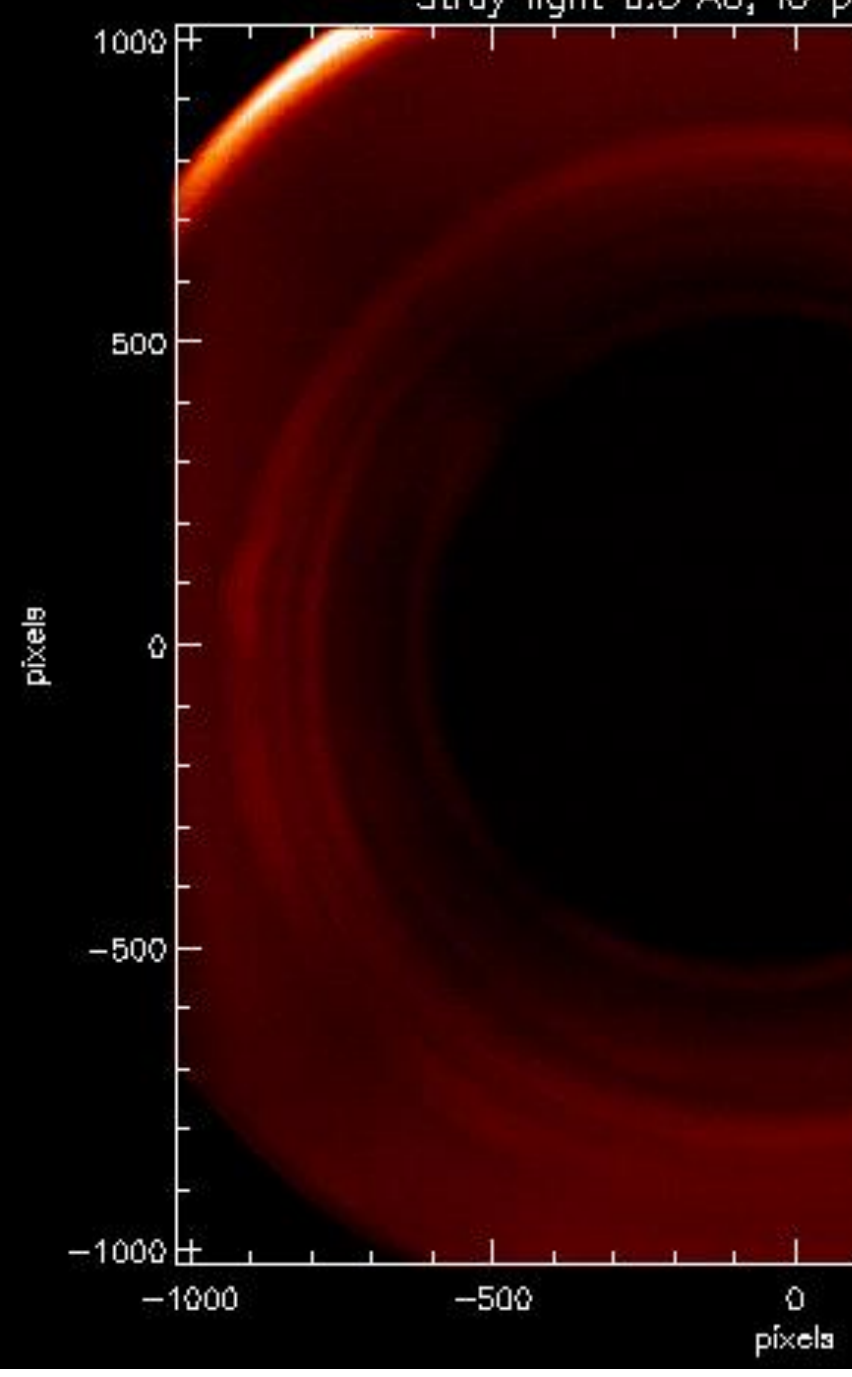

$10 / 10 / 2018$

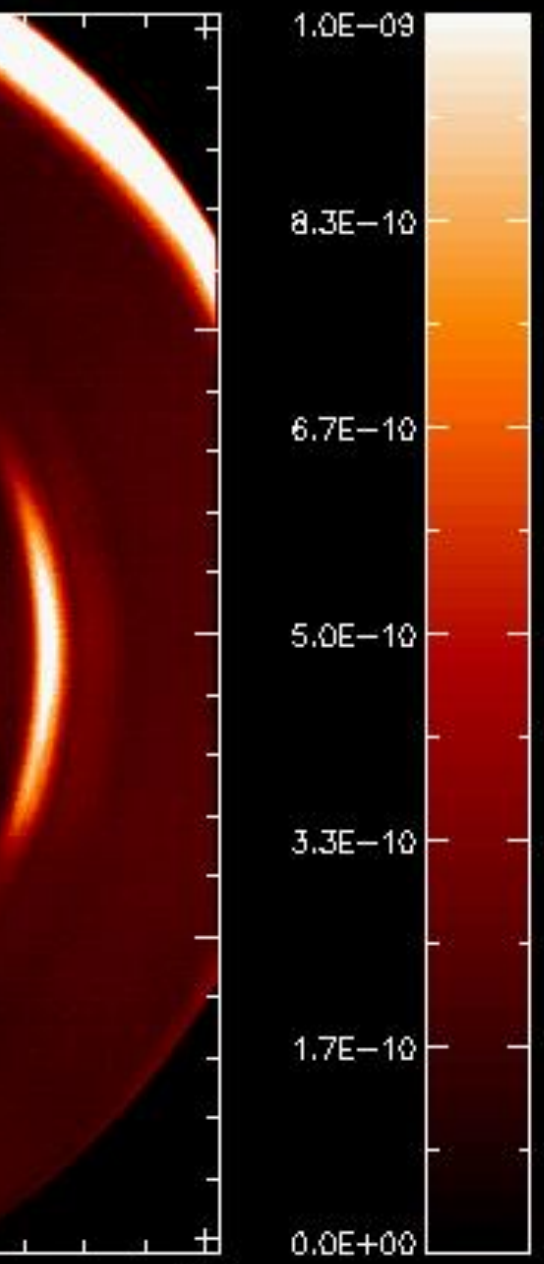

ए人

ICSO 2018 Chania (Greece) 


\section{D CAD simulation}

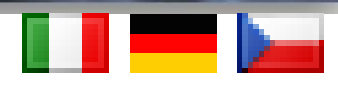

- We used the SPOCC 3D CAD and we took a simulated image with a camera replicating the same optical characteristics of Metis.
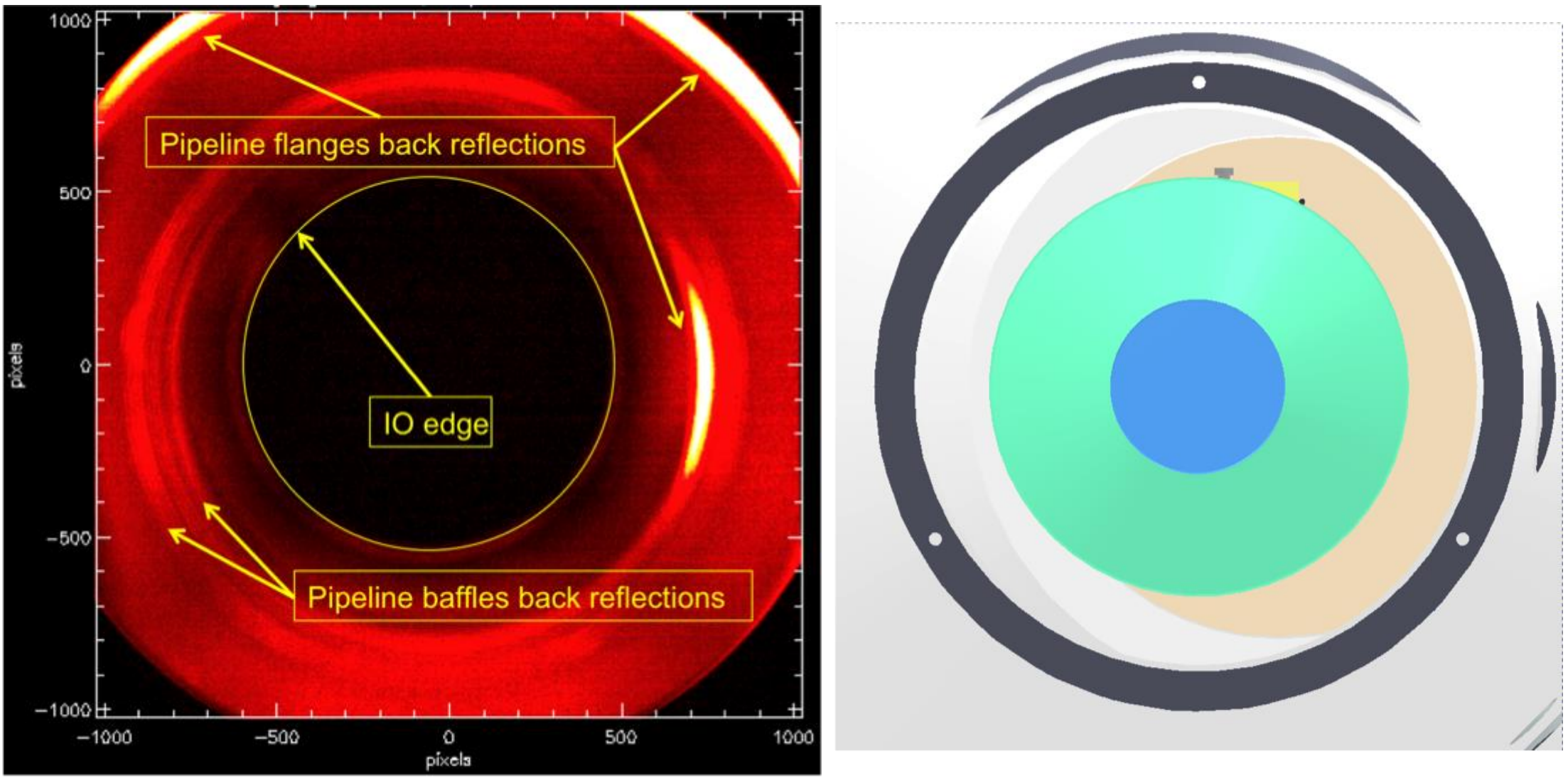


\section{Stray light 1.0 AU}

0

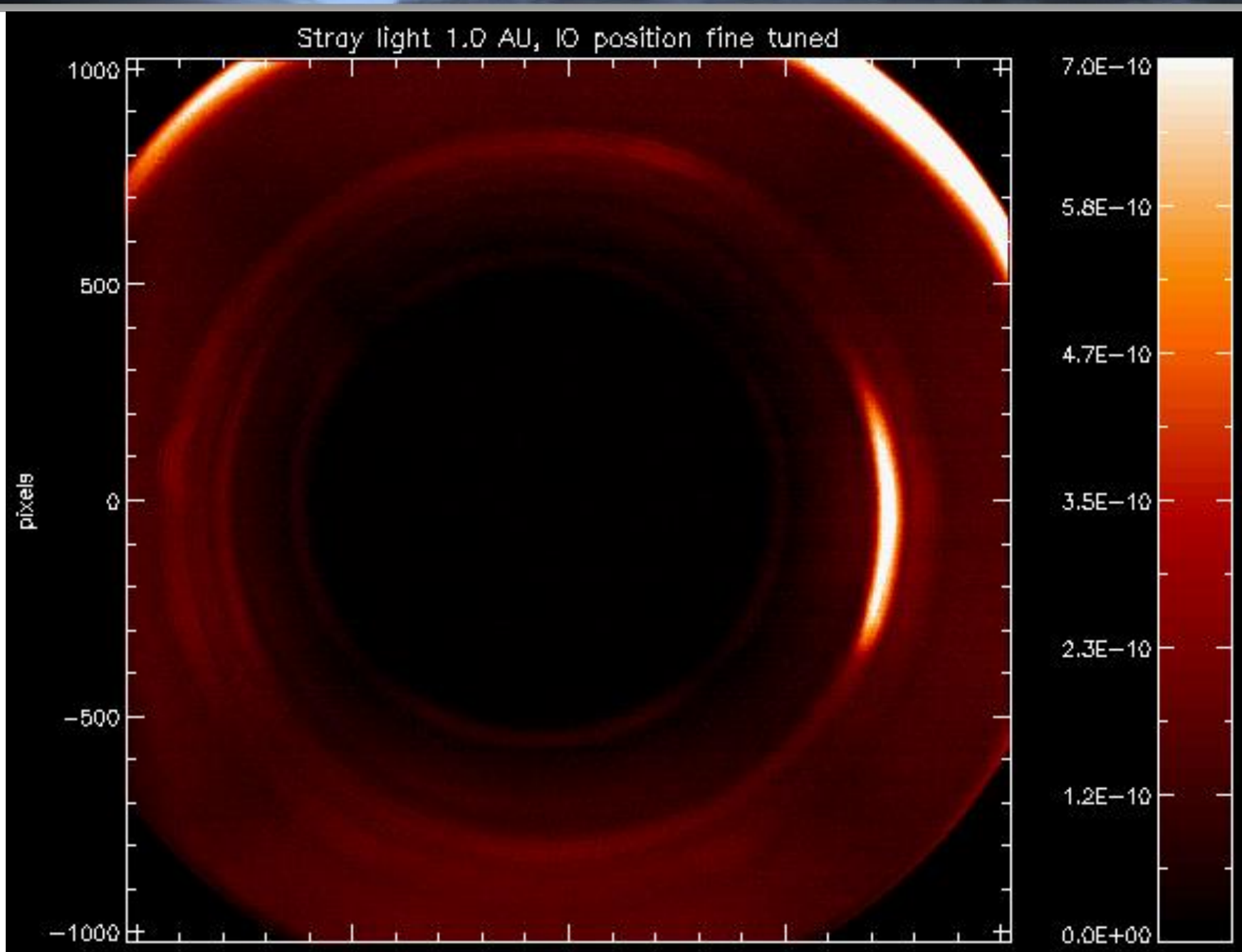




\section{Off pointing}

- Metis may experience off-pointings, due to spacecraft misalignments or to commanded operations.

- The SPOCC bench is motorized and was used to off-point Metis at $-0.6^{\circ},-$ $0.3^{\circ}, 0.3^{\circ}, 0.6^{\circ}$ in yaw.

- The signal average was evaluated in some ROls for each off-pointing.

- To avoid the influence of the setup, only 4 ROls were actually selected.

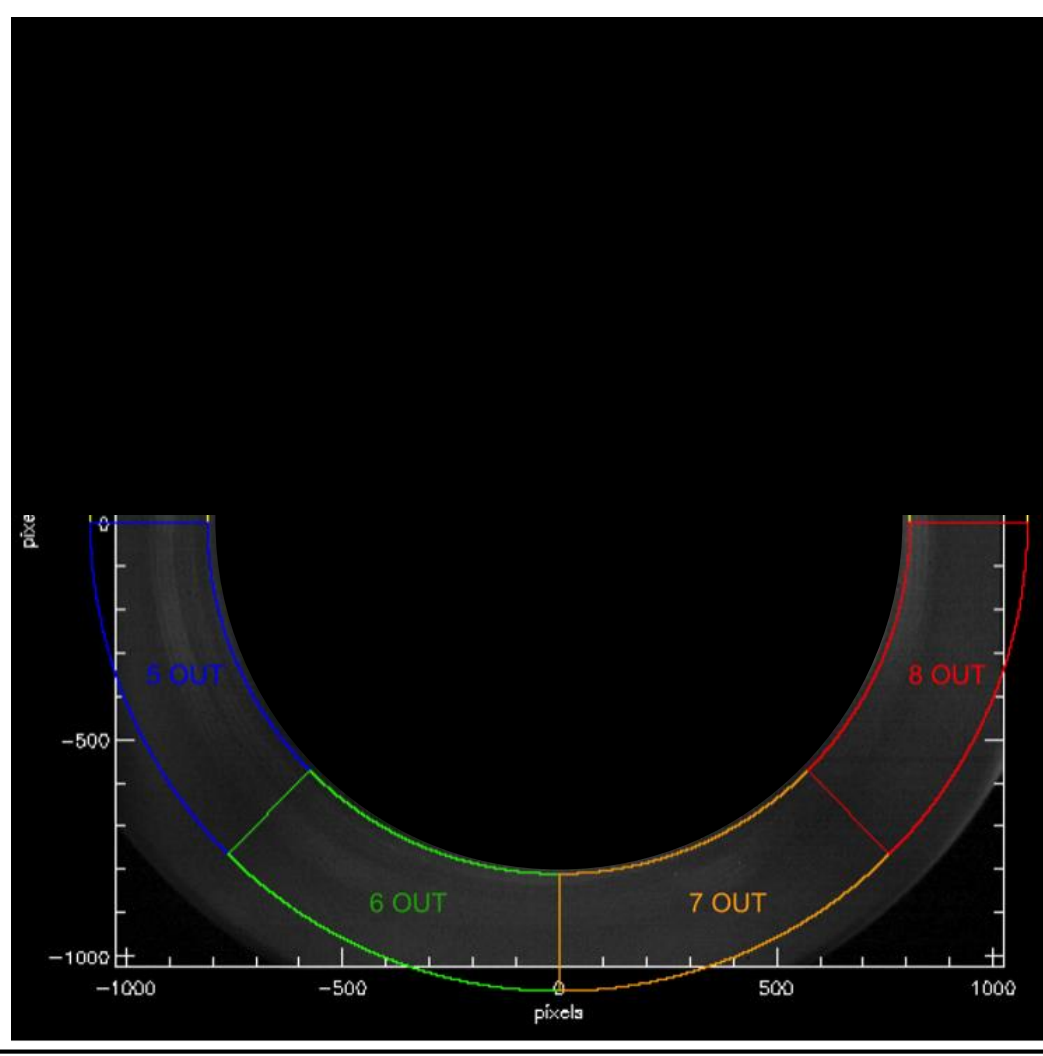




\section{Off-pointing}

- It is actually possible to use the method in flight to monitor the spacecraft offpointing.

- Refinements are necessary!

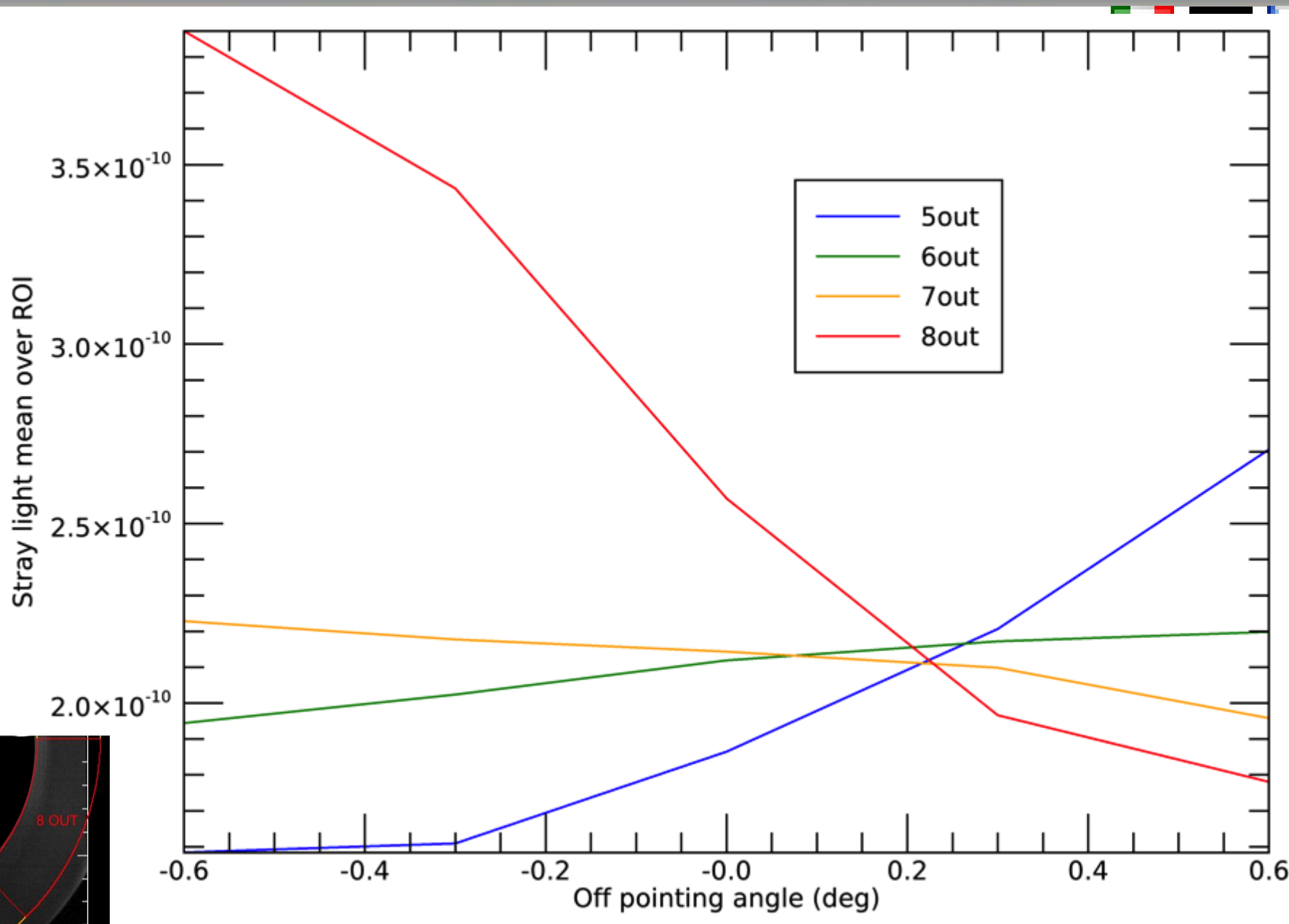




\section{Summary}

- Stray light calibration for the Solar Orbiter/Metis coronagraph were performed at the OPSys/SPOCC facility in Torino (Italy).

- After a fine tuning of the IO position, the stray light level matches the requirement (small deviations are due to light leaks in the setup).

- Stray light images were taken at 0.5 AU and 1.0 AU. As expected, the results are proportional to the solar disk dimension.

- An off-pointing investigation was performed as well: with opportune refinements, the diagnostic method can be used in flight to monitor the $\mathrm{S} / \mathrm{C}$ off-pointings by analyzing the stray light level.

- Metis is installed on the Solar Orbiter S/C and is about to undergo environmental tests.

- Launch scheduled in February 2020. 


\section{Acknowledgments}

- This work has been supported by the Italian Space Agency (ASI).

- OHB Italia (Prime Industrial Contractor towards ASI).

- Thales Alenia Space Italia (Co-Prime Industrial Contractor).

- ALTEC Company, sub-contractor, hosts the OPSys facility.

- The VLDA and UVDA assemblies were provided by MPS.

- The mirror hardware development was possible thanks to the Czech PRODEX Programme.

\section{Thank you for your attention}

